ARTICLE

\title{
An intrinsic timer specifies distal structures of the vertebrate limb
}

\author{
Patricia Saiz-Lopez ${ }^{1, \star}$, Kavitha Chinnaiya ${ }^{2, \star}$, Victor M. Campa ${ }^{1}$ Irene Delgado ${ }^{1, \dagger}$, \\ Maria A. Ros ${ }^{1,3} \&$ Matthew Towers ${ }^{2}$
}

How the positional values along the proximo-distal axis (stylopod-zeugopod-autopod) of the limb are specified is intensely debated. Early work suggested that cells intrinsically change their proximo-distal positional values by measuring time. Recently, however, it is suggested that instructive extrinsic signals from the trunk and apical ectodermal ridge specify the stylopod and zeugopod/autopod, respectively. Here, we show that the zeugopod and autopod are specified by an intrinsic timing mechanism. By grafting green fluorescent proteinexpressing cells from early to late chick wing buds, we demonstrate that distal mesenchyme cells intrinsically time Hoxa13 expression, cell cycle parameters and the duration of the overlying apical ectodermal ridge. In addition, we reveal that cell affinities intrinsically change in the distal mesenchyme, which we suggest results in a gradient of positional values along the proximo-distal axis. We propose a complete model in which a switch from extrinsic signalling to intrinsic timing patterns the vertebrate limb.

\footnotetext{
${ }^{1}$ Instituto de Biomedicina y Biotecnología de Cantabria, IBBTEC (CSIC-Universidad de Cantabria), Santander 39011, Spain. ${ }^{2}$ Bateson Centre, Department of Biomedical Sciences, University of Sheffield, Western Bank, Sheffield S10 2TN, UK. ${ }^{3}$ Departamento de Anatomía y Biología Celular, Facultad de Medicina, Universidad de Cantabria, Santander 39011, Spain. ${ }^{*}$ These authors contributed equally to this work. $\dagger$ Present address: Departamento de Desarrollo y Reparación Cardiovascular, Centro Nacional de Investigaciones Cardiovasculares, CNIC. 28029 Madrid, Spain. Correspondence and requests for materials should be addressed to M.A.R. (email: marian.ros@unican.es) or to M.T. (email: m.towers@sheffield.ac.uk).
} 
A $\mathrm{n}$ enigmatic problem in developmental biology is how the positional values along the proximo-distal axis (that is, humerus to digits) of the vertebrate limb are specified. Although this has been a topic of intense investigation, a consensus model has not been reached. Currently, a variation on a two-signal model involving proximal signals from the trunk and distal signals from the apical ectodermal ridge (AER) - the thickened epithelium that rims the distal mesenchyme of the limb-is favoured. Mercader et al. ${ }^{1}$ proposed a model in which AER-derived fibroblast growth factors (FGFs) repress a proximal programme that specifies the stylopod (that is, humerus), leaving it unclear as to how the more distal zeugopod (that is, radius/ ulna) and autopod (that is, wrist/digits) structures are specified. Instead, Mariani et al. ${ }^{2}$ suggested that instructive FGF signals specify presumptive autopod structures early, and that then intercalation between this domain and the presumptive stylopod results in the specification of the zeugopod. However, this sequence of proximo-distal specification has not been confirmed, as no molecular markers are known. Recently, work on the chick limb has shown that the stylopod is specified by diffusible signals, the identity of which is considered to be retinoic acid ${ }^{3,4}$, although this is controversial ${ }^{5}$. Therefore, how the zeugopod and autopod segments are specified remains contentious and some patterns of gene expression in the chick wing bud are not consistent with a signal-based mechanism. Thus, Hairy2 (a gene involved in the somitogenesis clock) displays an oscillatory expression pattern ${ }^{6}$ and Hoxa13 (an autopod marker), cannot be prematurely activated by AER signals that maintain its expression ${ }^{7,8}$. It has been proposed that Hoxa13 expression is more consistent with the earlier progress zone model ${ }^{9}$ (for recent discussions see refs $10,11)$. In the progress zone model, derived from embryological experiments on the chick wing bud, timing by an intrinsic clock operating in distal mesenchyme cells (the progress zone) is proposed to specify proximo-distal positional values ${ }^{9}$. An additional feature of this model is that extrinsic AER signals give distal mesenchyme cells the competence to measure time according to their own intrinsic clock.

To distinguish between intrinsic and signal-based timing mechanisms for specifying the zeugopod and autopod segments, we performed heterochronic transplants of small blocks of distal mesenchyme cells $(150 \mu \mathrm{m})$ from young green fluorescent protein (GFP)-expressing ${ }^{12}$ donor chick wing buds underneath the intact AER of older wild-type host wing buds: a procedure that preserves the host limb bud architecture and incorporates the donor tissue within the signalling environment of the host distal mesenchyme. It is predicted that, if proximo-distal specification is intrinsically timed, then grafts of $\mathrm{HH} 20$ distal cells made to older (by $24 \mathrm{~h}$ ) HH24 buds should behave according to the age of the donor wing bud; but if fate is controlled by extrinsic signalling, then the grafted cells should be re-specified and develop according to the host age. Using this technique, we reveal that distal cells develop according to their own age and that the positional values of the zeugopod and autopod are progressively specified in an intrinsically timed manner.

\section{Results}

The host environment appears to determine distal graft fate. Earlier dye-based fate maps revealed that $\mathrm{HH} 20$ cells at the distal mesenchyme of the chick wing give rise to the zeugopod (radius/ ulna), and at HH24, the autopod (wrist/digits) ${ }^{13,14}$. To see if our grafting technique could replicate these earlier fate maps, we performed homochronic grafts of $150 \mu \mathrm{m}$ blocks of HH20 and HH24 GFP-expressing chick wing distal tissue under the AER of wild-type host buds (Fig. 1a,d). On days 11-12 of development, the GFP-labelled cells gave rise to the same structures along the proximo-distal axis as mesenchyme cells labelled at equivalent stages with dyes ${ }^{13,14}$-zeugopod and autopod with $\mathrm{HH} 20$ grafts (Fig. 1b,c-blue asterisks, j) and autopod only with HH24 grafts (Fig. 1e,f-red asterisks, j). These fate maps show that grafts of distal mesenchyme cells incorporate well and develop like host tissue. To understand if extrinsic signals or an intrinsic timing mechanism determines the proximo-distal positional values of grafted cells, and hence the structures that they contribute to, we made heterochronic grafts of blocks of HH20 distal tissue to HH24 buds (Fig. 1g). Analyses of the fate maps on days 11-12 revealed that these grafts gave rise to structures distal to the zeugopod (Fig. 1h,i-black asterisks, j) comparable with the presumptive fate of host $\mathrm{HH} 24$ distal cells and not donor HH20 cells (Fig. 1j). Therefore, this finding suggests that the signalling environment provided by the age of the host determines the positional value of grafted chick wing distal mesenchyme cells.

Hoxa13 is intrinsically timed in distal cells. Previous evidence suggests that AER-derived FGFs in the chick wing bud are required to remove retinoic acid signals from the flank that

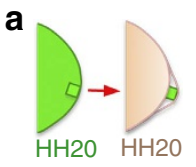

d

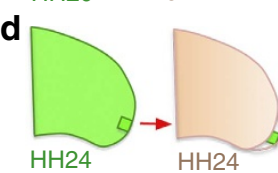

g

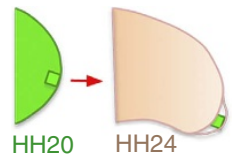

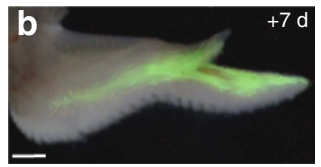

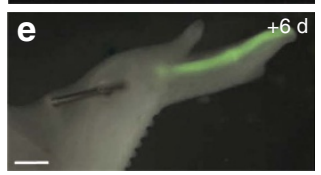

h

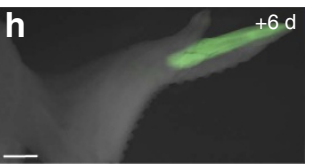

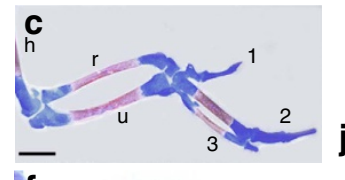

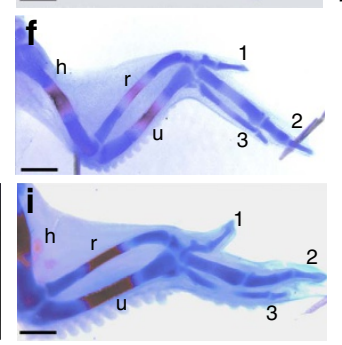

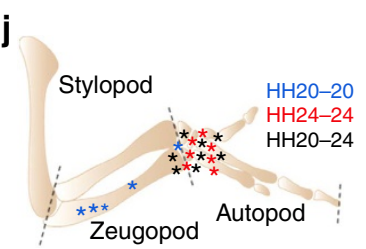
(1)

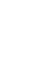

Figure 1 | The environment appears to determine distal graft fate. Blocks $(150 \mu \mathrm{m})$ of GFP-expressing (depicted as green in this and all schematic

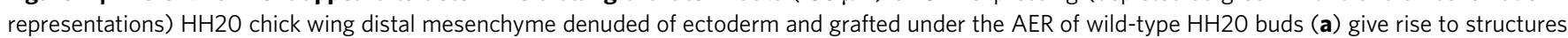

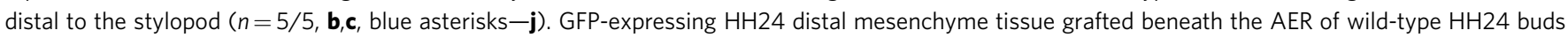

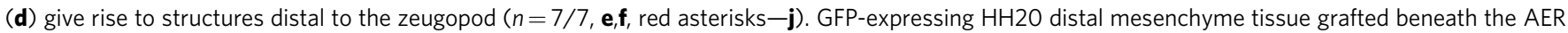

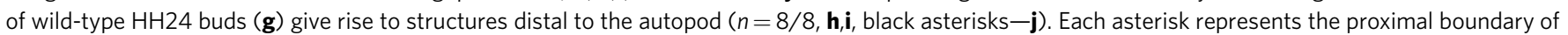
the grafted tissue for each experiment. Note, h-humerus; u-ulna; r-radius; 1,2 and 3 are the digits in anterior to posterior sequence. Scale bars: 1 mm. 
inhibit the epigenetically timed programme of expression of the distal autopod marker, Hoxa13 (refs 7,8) (see normal expression pattern-Supplementary Fig. 1). Therefore, this is not consistent with the positional values of distal mesenchyme cells being determined by the age of extrinsic signalling environment (Fig. 1g-j). To address this discrepancy, we asked whether, in our heterochronic grafting assay, Hoxa13 expression reflects the age of the donor distal mesenchyme or the host-signalling environment. In control homochronic grafts of HH20 distal mesenchyme cells made to HH20 buds (Fig. 2a), activation of Hoxa13 expression occurred concomitantly in host and donor tissue and a normal pattern was observed after $24 \mathrm{~h}$, showing that the grafting process does not affect the dynamics of Hoxal3 transcription (Fig. 2b,c, note serial sections hybridized with Hoxa13 and Gfp riboprobes to show the graft). However, in heterochronic $\mathrm{HH} 20$ grafts made to HH24 buds (Fig. 2d), Hoxa13 expression in the graft was undetectable until around $24 \mathrm{~h}$ after grafting (Fig. 2e,f). In fact, transcripts were first detected in the distal part of the grafted tissue (arrows-Fig. 2e,f-then at HH24) and were absent in the proximal part (asterisks Fig. 2e,f), despite the entire graft being surrounded by Hoxa13-expressing host tissue (then at HH27-see also Supplementary Fig. 2). By $48 \mathrm{~h}$, Hoxal 3 was expressed throughout the graft (then at HH27) and was indistinguishable from host tissue expression (then at HH29, Fig. 2g,h). Together, these data show that, despite the age of the extrinsic environment appearing to dictate the proximo-distal level that heterochronic grafts contribute to (Fig. 1), the activation of Hoxal3 expression is timed on donor schedule. In addition, our results confirm that AER signals, either directly or indirectly, are required for Hoxal3 induction, since grafts made to the presumptive zeugopod do not express Hoxa13 (Supplementary Fig. 3). In addition, the rapid proximal spread of Hoxal3 expression within the graft suggests that the establishment of the mature Hoxa13 expression domain (Supplementary Fig. 1) is not just due to proliferation of a small founder population of sub-AER HH22 distal mesenchyme cells ${ }^{15}$, but also due to transcriptional initiation in progressively more proximal tissue. An alternative explanation for the distal restriction of Hoxa13 expression in heterochronic grafts is that more proximal cells selectively die, but TUNEL analyses revealed no evidence of abnormal apoptosis (Supplementary Fig. 2).
AER duration is timed by the distal mesenchyme. The AER is maintained by signalling from the underlying mesenchyme ${ }^{16,17}$ and is required for limb outgrowth until it regresses at around $\mathrm{HH} 29 / 30$ in the chick wing ${ }^{18}$. Therefore, since Hoxa13 expression is intrinsically timed, we asked if the capacity to maintain the AER is also an intrinsic property related to the age of the distal mesenchyme, by examining Fgf8 expression. During normal development, high-level Fgf8 expression abruptly terminates at around stage $\mathrm{HH} 29$, first in the AER over the interdigits and then over the digits ${ }^{19}$. In control homochronic grafts of $\mathrm{HH} 24$ distal mesenchyme cells made to HH24 buds (Fig. 3a), after $48 \mathrm{~h}$ (HH29), Fgf8 expression was indistinguishable between righthand experimental and left-hand contralateral buds (asterisks, Fig. 3b-d). At 72 h (HH30), only residual Fgf8 expression could be detected in left and right buds, showing that transcription was terminated at the same time (asterisks, Fig. 3e-g). Instead, in heterochronic grafts of HH20 distal tissue made to $\mathrm{HH} 24$ host buds (Fig. 3h), after 48 and $72 \mathrm{~h}, F g f 8$ was expressed in the AER overlying the grafted tissue at higher levels than in the equivalent AER region of the contralateral bud (asterisks, Fig. 3i-n). Therefore, the expression of Fgf8 in the AER over the graft was extended for about $24 \mathrm{~h}$ longer than in the corresponding region of contralateral limb AER-equivalent to the difference in age between host and donor tissue. This result demonstrates that distal mesenchyme cells can locally maintain the AER in an intrinsically timed manner.

Distal cell cycle parameters are intrinsically timed. We have previously shown that the intrinsic behaviour of Sonic hedgehogproducing polarizing region cells of the chick wing bud is linked with stage-specific cell cycle parameters ${ }^{20}$. Therefore, to test if adjacent non-polarizing distal mesenchyme cells have stagespecific cell cycle parameters, we undertook flow cytometric analyses at a range of stages between $\mathrm{HH} 20$ and $\mathrm{HH} 30$ (equivalent to the distal $150 \mu \mathrm{m}$ tissue used in grafting experiments). We found that the proportion of chick wing distal mesenchyme cells in G1-phase increases from 56.9 to $64.5 \%$ between stages $\mathrm{HH} 20$ and $\mathrm{HH} 27$ ( 48 h), then abruptly to $85.7 \%$ by HH30 ( $\sim 96 \mathrm{~h}$ after $\mathrm{HH} 20$ ) indicating a loss of proliferative potential (Fig. 4a) that is comparable with the cells of the polarizing region ${ }^{20}$. Accordingly, the proportion of S-phase cells
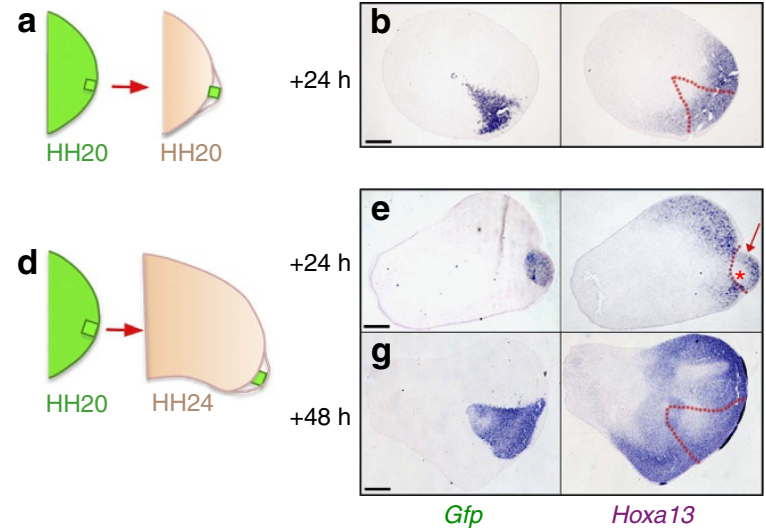
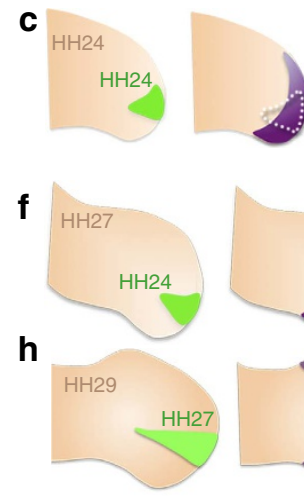

Gfp

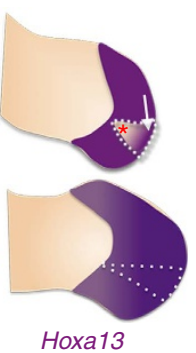

Figure 2 | Hoxa13 expression is intrinsically timed. In grafts of $\mathrm{HH} 20$ distal mesenchyme cells made to the same stage wing buds (a), the presence of the grafted tissue does not perturb the establishment of a normal domain of Hoxa13 expression. Note that the graft cannot be distinguished by Hoxa13 expression at $24 \mathrm{~h}$. (b,c) $n=2 / 2$ - note area of grafted tissue is shown by Gfp expression in a serial section (b) and dashed lines. In grafts of GFPexpressing $\mathrm{HH} 20$ distal mesenchyme tissue grafted beneath the AER of wild-type HH24 buds (d) Hoxa13 is expressed in an intrinsically timed manner shown $24 \mathrm{~h}(\mathbf{e}, \mathbf{f}-n=3 / 3)$ and $48 \mathrm{~h}$ after grafting $(\mathbf{g}, \mathbf{h}-n=3 / 3)$. Note that the arrows in (e,f) indicate Hoxa13 expression in distal part of graft and asterisk absence in proximal part of the graft. Scale bars: $100 \mu \mathrm{m}$. 


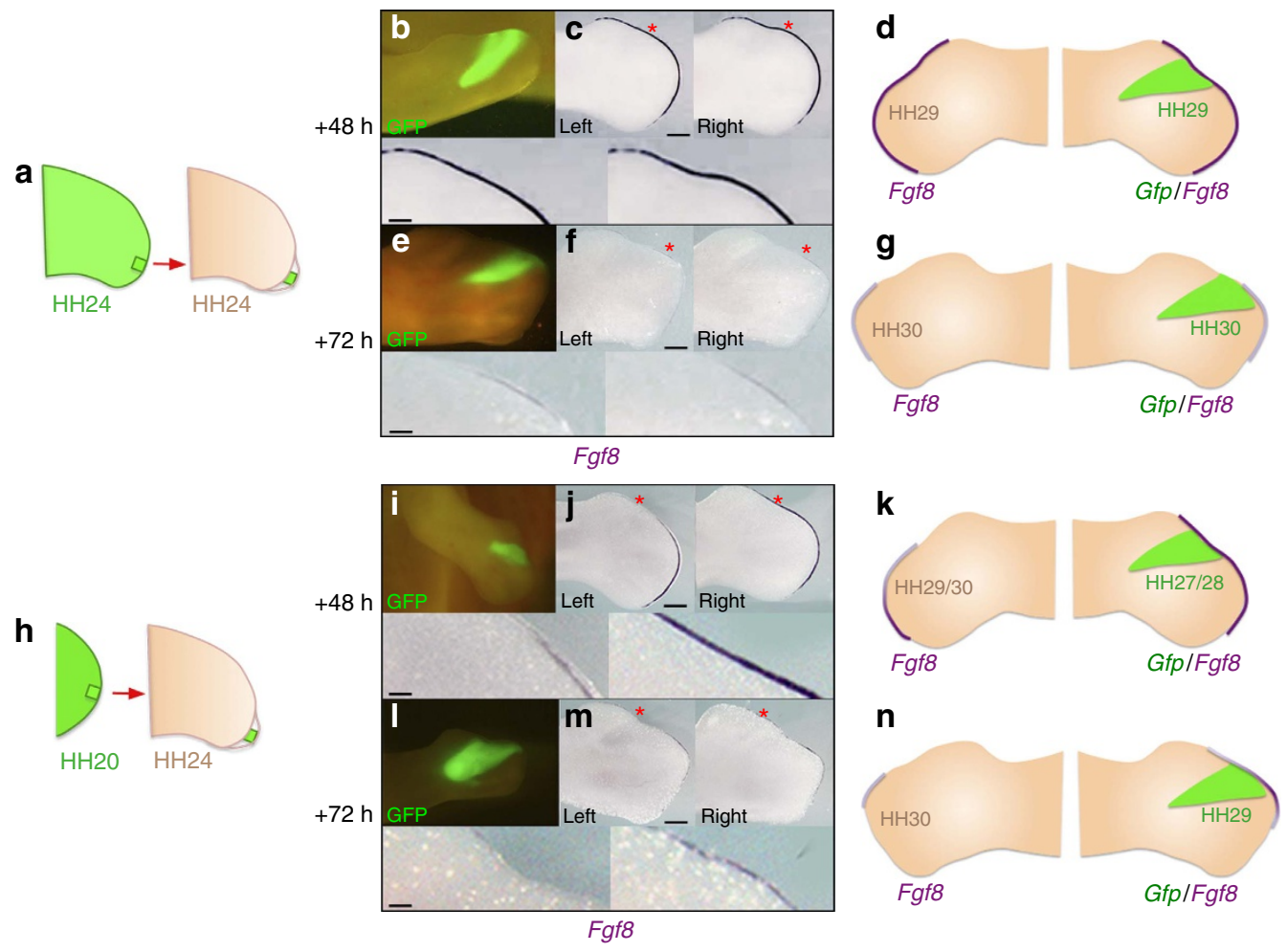

Figure 3 | AER duration is locally controlled by the distal mesenchyme. In grafts of $\mathrm{HH} 24$ distal mesenchyme cells made to the same stage wing buds (a), the pattern of Fgf8 expression in the AER is indistinguishable between left and right buds after $48 \mathrm{~h}(\mathbf{b}-\mathbf{d}, n=2 / 2)$ and $72 \mathrm{~h}(\mathbf{e}-\mathbf{g} n=2 / 2)$. Note area of grafted tissue is shown by GFP expression (b,e) and lower panels are higher magnifications of areas marked with asterisks (c,f). In grafts of GFP-expressing $\mathrm{HH} 20$ distal mesenchyme tissue grafted beneath the AER of wild-type $\mathrm{HH} 24$ buds (h) Fgf8 is stronger in manipulated right buds compared with equivalent region of contralateral left buds after $48 \mathrm{~h}(\mathbf{i}-\mathbf{k}, n=2 / 2)$ and $72 \mathrm{~h}(\mathbf{I}-\mathbf{n}, n=2 / 2)$. Note area of grafted tissue is shown by GFP expression (i,I) and lower panels are higher magnifications of areas marked with asterisks $(\mathbf{j}, \mathbf{m})$. Left limb photos flipped horizontally for comparison in (c,f,j, $\mathbf{m})$. Scale bars in $(\mathbf{c}, \mathbf{f}, \mathbf{j}, \mathbf{m})-100 \mu \mathrm{m}$ and in their enlarged panels beneath $-25 \mu \mathrm{m}$.

decreases from 18.0 to $4.4 \%$ and G2/M-phase cells decreases from 25.1 to $9.9 \%$ over the same time interval (Fig. 4 a). To examine whether, as with the polarizing region, the cell cycle parameters of the adjacent distal mesenchyme are intrinsically controlled, we carried out flow cytometry on cells from grafts of $\mathrm{HH} 20$ distal tissue made under the AER of HH24 buds (Fig. 4b). By 48 (host at $\mathrm{HH} 29$ ) and $72 \mathrm{~h}$ (host at $\mathrm{HH} 30$ ), the proportion of G1-phase cells in the distal most $150 \mu \mathrm{m}$ of the grafted tissue was significantly less (6.2 and 7.3\% reduction, respectively) than in equivalent host tissue in contralateral buds (Fig. 4c,d, G1-phase percentages differ by $<2 \%$ in left and right distal mesenchyme cells of normal buds and also those with homochronic grafts, see Supplementary Tables 1 and 2). In addition, the proportion of cells in S and G2/M phases in grafted tissue was significantly higher than in host tissue, consistent with increased proliferative potential (Fig. 4c,d). Indeed, the cell cycle phase values of grafted cells are similar to those expected for the stages of the younger donor embryos (HH27 and HH29-Fig. 4a). These data show that the cell cycle parameters of chick wing distal mesenchyme cells are intrinsically regulated.

Distal positional values are intrinsically timed. Having demonstrated that grafts of $\mathrm{HH} 20$ distal mesenchyme cells made to HH24 buds intrinsically time Hoxa13 expression, AER maintenance and cell proliferation, we investigated why their proximodistal positional value appears to be influenced by the age of the host environment (Fig. 1h-j). Positional values are expressed as a gradient of cell adhesion along the proximo-distal axis of the $\operatorname{limb}^{21}$ and the differential adhesion between proximal and distal chick wing bud cells causes them to sort out in culture ${ }^{22,23}$. Therefore, we tested if the adhesive properties, and hence positional values of distal mesenchyme cells, are acquired in response to extrinsic signals or by an intrinsic timing mechanism in vivo. To achieve this, we disaggregated blocks of wild-type HH20 (zeugopod progenitor-Fig. 1j) and GFP-expressing HH24 (autopod progenitor-Fig. 1j) distal mesenchyme cells into single cells that were then re-aggregated and grafted to HH24 buds (see Materials and methods). Thus, if positional values are specified by host $\mathrm{HH} 24$ signals, then the grafted $\mathrm{HH} 20$ and $\mathrm{HH} 24$ cells are predicted to acquire equivalent adhesive properties and remain randomly dispersed in the grafts. Alternatively, if positional values are specified by an intrinsic timer, then grafted $\mathrm{HH} 20$ cells are not expected to be influenced by host $\mathrm{HH} 24$ signals, so should maintain adhesive properties reflective of their programmed fate, and thus sort out from grafted $\mathrm{HH} 24$ cells.

In control grafts of GFP-expressing $\mathrm{HH} 24$ and wild-type $\mathrm{HH} 24$ distal mesenchyme cells made to HH24 buds (Fig. 5a), there appeared to be a random distribution of cells as assessed by GFP immunofluorescence (Fig. 5b-e-see Supplementary Fig. 4 for detection of Gfp mRNA). To quantitate this behaviour, we determined the distribution of GFP-expressing cells between the inner and outer regions of such grafts (inner/outer defined as half the distance from the centre of the graft to the periphery-see Materials and methods). At $24 \mathrm{~h}$, the inner/outer distribution of grafted GFP-expressing $\mathrm{HH} 24$ cells was $49 \% / 51 \%$ (Fig. 5b,c) and at $48 \mathrm{~h}, 55 \% / 45 \%$ (Fig. $5 \mathrm{~d}, \mathrm{e}$ ). On the other hand, grafts of GFPexpressing $\mathrm{HH} 24$ cells and wild-type $\mathrm{HH} 20$ cells made to $\mathrm{HH} 24$ 

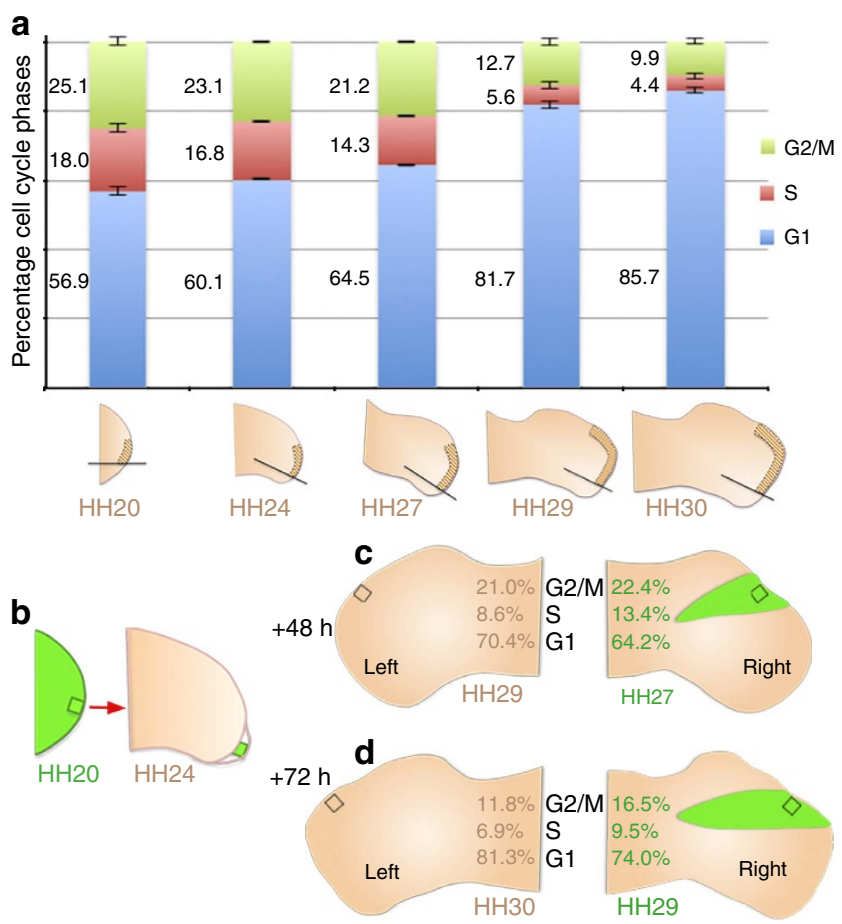

Figure 4 | Cell cycle parameters are intrinsically timed. Cell cycle

parameters of $\mathrm{HH} 2 \mathrm{O}$ (three repeat experiments to account for variation in staging), $\mathrm{HH} 24$ (6), $\mathrm{HH} 27$ (6), $\mathrm{HH} 29$ (2) and $\mathrm{HH} 30$ (5) distal mesenchyme tissue-bars indicate standard error of the mean between the repeat experiments (a). In each experiment between 8,000 and 10,000 cells were counted from 10 to 12 pooled strips of distal tissue each obtained from separate buds. Blocks $(150 \mu \mathrm{m})$ of GFP-expressing $\mathrm{HH} 20$ distal mesenchyme tissue grafted beneath the AER of wild-type $\mathrm{HH} 24$ buds (b), after $48 \mathrm{~h}$ (c), $\mathrm{G} 1=64.2 \%, \mathrm{~S}=13.4 \%, \mathrm{G} 2 / \mathrm{M}=22.4 \%$ phase cells in grafted right wing distal mesenchyme blocks $(150 \mu \mathrm{m})$ compared with $\mathrm{G} 1=70.4 \%, \mathrm{~S}=8.6 \%, \mathrm{G} 2 / \mathrm{M}=21.0 \%$ phase cells in equal numbers of contralateral left wing bud distal mesenchyme blocks (one pool of $n=10$ cubes of tissue, each taken from separate left control and experimental right buds). After $72 \mathrm{~h}$ (d), G1 $=74.0 \%, \mathrm{~S}=9.5 \%, \mathrm{G} 2 / \mathrm{M}=16.5 \%$ of cells in grafted right wing distal mesenchyme blocks $(150 \mu \mathrm{m})$ compared with $\mathrm{G} 1=81.3 \%, \mathrm{~S}=6.9 \%, \mathrm{G} 2 / \mathrm{M}=11.8 \%$ of cells in equal numbers of contralateral left wing bud distal mesenchyme blocks (one pool of $n=10$ cubes of tissue in both cases). In both experiments, more than 10,000 cells were counted and there is a significant difference in $\mathrm{G} 1, \mathrm{~S}$ and $\mathrm{G} 2 / \mathrm{M}$ numbers between left and right distal mesenchyme (Pearson's $\chi^{2}$ test$P<0.0001)$ consistent with graft behaving intrinsically.

buds (Fig. 5f) showed a non-random distribution (Fig. 5g-j, see Supplementary Fig. 4 for detection of Gfp mRNA). At $24 \mathrm{~h}$, the inner/outer distribution of grafted GFP-expressing HH24 cells was $24 \% / 76 \%$ (Fig. 5g,h) and at $48 \mathrm{~h}, 29 \% / 71 \%$ (Fig. 5i,j). Therefore, this result shows that GFP-expressing HH24 distal mesenchyme cells had sorted out to the periphery of the graft, to associate with host tissue of the same age (then at HH27, Fig. 5g,h and $\mathrm{HH} 29$, Fig. 5i,j), In addition, this cell sorting confines wildtype $\mathrm{HH} 20$ distal mesenchyme cells (then at HH24, Fig. 5g,h and $\mathrm{HH} 27$, Fig. 5i,j) to the centre of the grafts (Supplementary Fig. 4). A similar pattern was still observed in 12-day-old wings thus showing that the distribution of cells caused by this earlier sorting event is maintained. Therefore, grafted GFP-expressing HH24 cells contact host cells of the same age (then both at day 12) and surround grafted HH20 wild-type cells (then at day 11, Fig. $5 \mathrm{k}-\mathrm{n}$ ). This result suggests that the inability of grafted HH20 zeugopod progenitor cells to contact host cells with equivalent positional values - that would have been displaced proximally at an earlier stage-entrains them into autopod structures and explains why grafts comprising $\mathrm{HH} 20$ cells made to $\mathrm{HH} 24$ buds are only able to contribute to the autopod (Fig. 5o-see Fig. 1h-j).

In summary, these data show that individual cells of different ages and proximo-distal fates sort out when grafted beneath the AER, thus indicating that the adhesive properties of cells, and hence positional values of the chick wing zeugopod and autopod, are specified by an intrinsic timer.

\section{Discussion}

In this study, we set out to determine the contributions that extrinsic signalling and intrinsic timing mechanisms play in the specification of the distal structures of the limb. Our results provide evidence that an intrinsic timer, operating in distal chick wing mesenchyme cells, specifies the positional values of the zeugopod and autopod-consistent with the classical progress zone model ${ }^{9}$. Our findings allow us to present a complete model of proximo-distal limb patterning (Fig. 6a-d).

During limb initiation stages, trunk-derived retinoic acid specifies the stylopod ${ }^{3,4}$ (Fig. 6a-HH18/19 in the chick wing characterized by Meis1/2 expression). Over time, AER-derived FGFs induce the retinoic acid-degrading enzyme Cyp26b1 (ref. 24), and this, along with limb bud outgrowth, creates a retinoic acid-free distal mesenchyme domain (Fig. 6b,c). We propose that this event triggers an intrinsic timer in distal mesenchyme cells and the switch from proximal (stylopod) to distal (zeugopod/autopod) specification. Distal mesenchyme cells that activate this programme first transit through a phase of zeugopod specification (Fig. 6b- $\mathrm{HH} 20-22$, characterized by Hoxa/d11 expression), and then a second phase of autopod specification (Fig. 6c-HH23-24, characterized by Hoxa/d13 expression).

Importantly, we demonstrated that this intrinsic programme results in the acquisition of stage-specific cell adhesion properties, which are a read-out of proximo-distal positional values ${ }^{21-23}$. The fact that cells are being constantly displaced from the distal mesenchyme by an intrinsically timed programme of proliferation thus provides a robust mechanism by which a precise gradient of positional values can become established (Fig. 6d). Our findings therefore support the early idea that an intrinsic cell cycle clock $^{25}$, sustained by AER signalling, is part of the timing mechanism that specifies the positional values of the zeugopod and autopod.

It has previously been shown that the switch from proximal to distal specification is associated with the epigenetic activation of the distal autopod marker, Hoxa13 (ref. 8). We have extended these studies in showing that timing of Hoxa13 expression is related to the age of the mesenchyme and is activated in a distal to proximal wave, which we suggest reflects the removal of inhibitory retinoic acid from the limb bud. Although it is unclear how the specification of positional values relates to the final limb anatomy, it is intriguing that Hoxa13 regulates cell adhesion molecules such as EphrinA7 (ref. 26). Therefore, given that the dosage of $5^{\prime} H o x a / d$ gene products modulates the number of elements forming in the autopod by a Turing-type reactiondiffusion mechanism ${ }^{27}$, it is tempting to speculate that a link between this process of self-organization and cell adhesion exists.

Our finding that the intrinsic timing mechanism is not affected by the age of the AER supports the early proposal that AER signalling in the chick wing fulfils a permissive role in proximodistal patterning (Fig. $6 \mathrm{a}-\mathrm{c})^{28}$, rather than the instructive role suggested by genetic experiments on the mouse limb ${ }^{2}$. Moreover, we showed that distal mesenchyme cells locally maintain the overlying AER for the appropriate duration to sustain outgrowth 

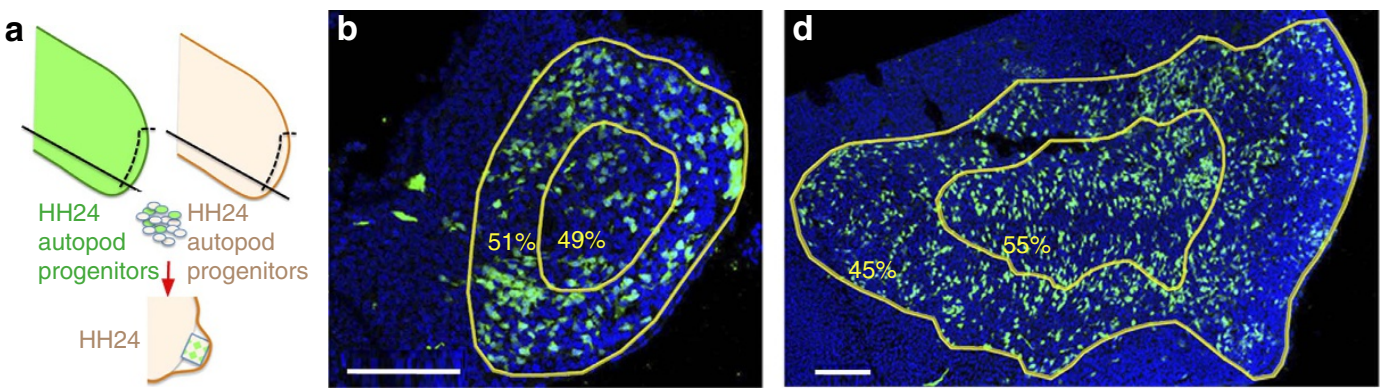

$+24 \mathrm{~h}$

GFP/DAPI

$+48 \mathrm{~h}$

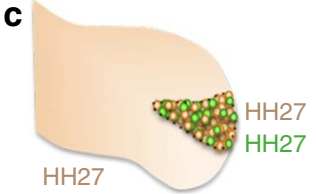

e
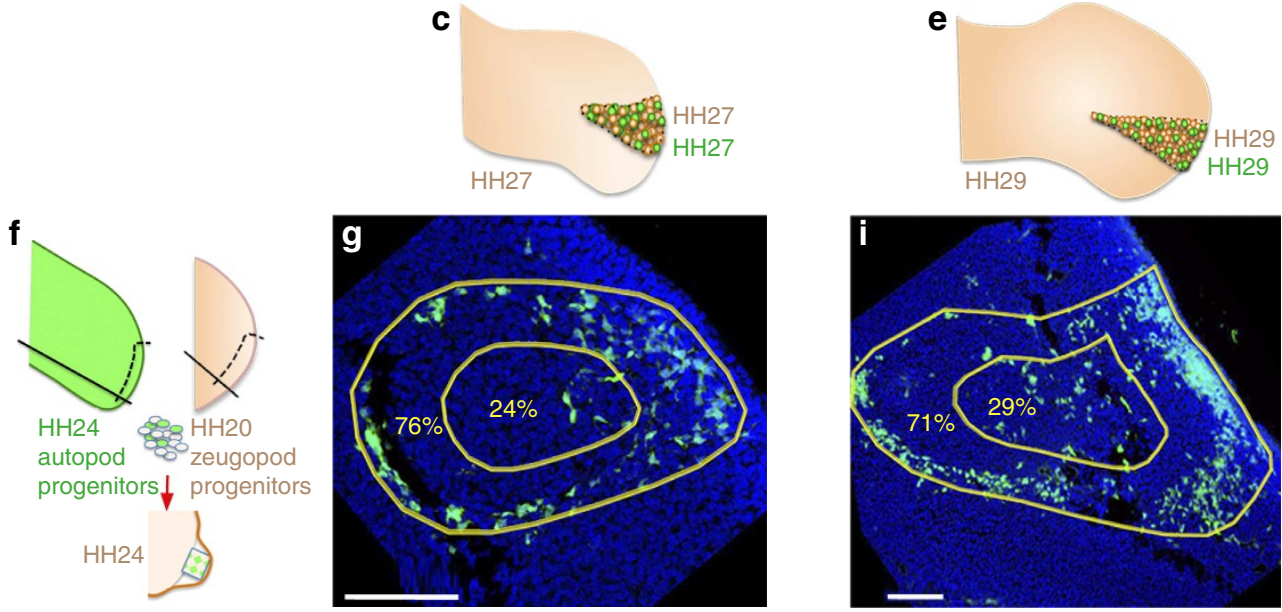

h

$+24 \mathrm{~h}$

GFP/DAPI

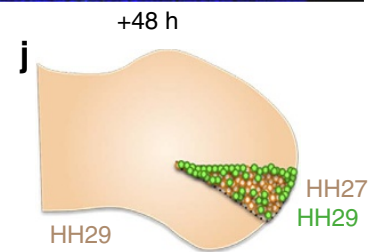

$\mathbf{k}$
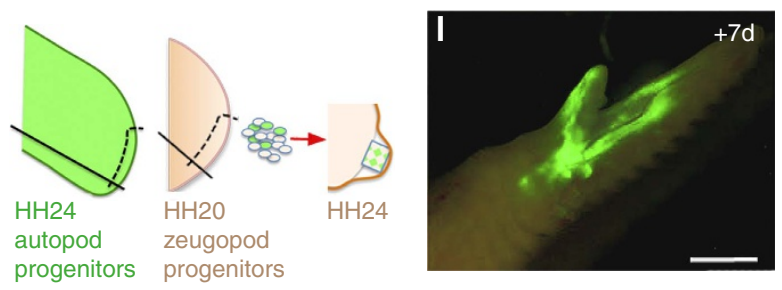

m
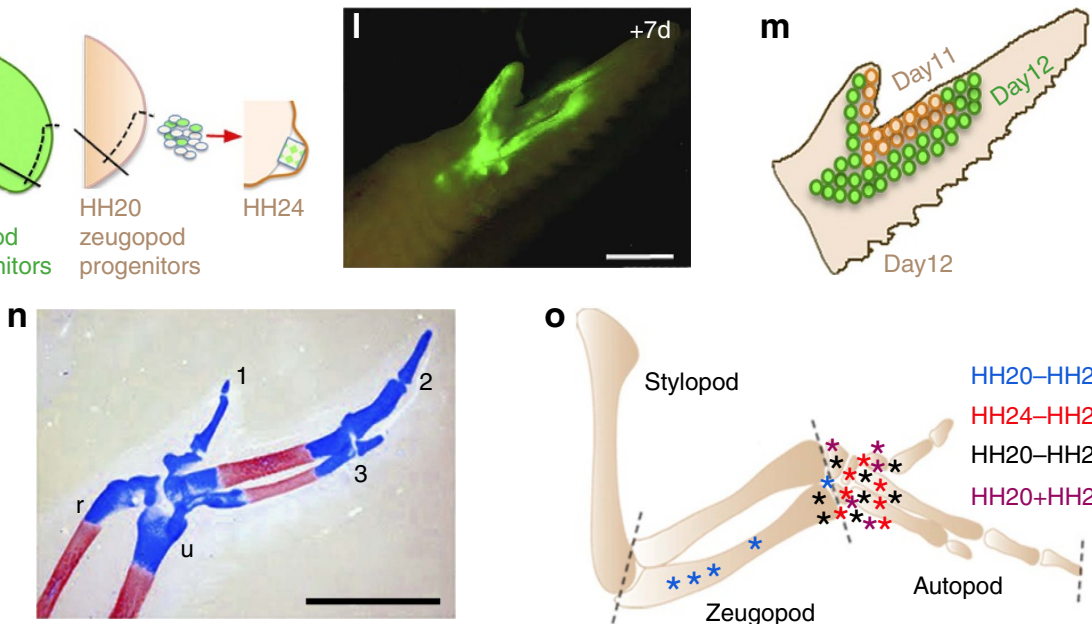

o

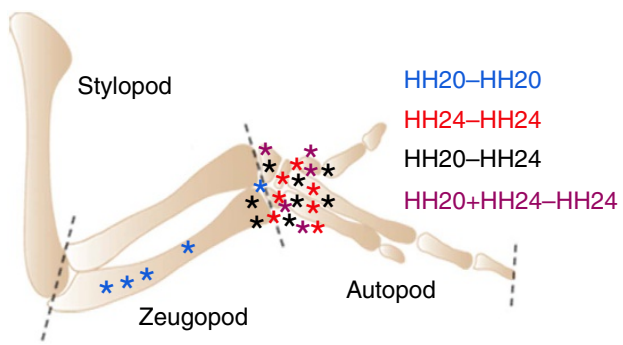

Figure 5 | An intrinsic timer specifies distal positional values. Disaggregated GFP-expressing $\mathrm{HH} 24$ and wild-type $\mathrm{HH} 24$ autopod progenitor cells re-aggregated into pellets and grafted to $\mathrm{HH} 24$ buds (a) show an approximately equal distribution in the inner and outer regions of grafts after 24 $(\mathbf{b}, \mathbf{c}, n=2 / 2)$ and $48 \mathrm{~h}(\mathbf{d}, \mathbf{e}, n=3 / 3$, green shows GFP immunofluorescence and blue DAPI staining-the inner/outer regions defined as half the distance from the centre of the graft to the periphery as indicated by yellow rings). Disaggregated HH24 GFP-expressing autopod progenitor cells and wild-type $\mathrm{HH} 20$ zeugopod progenitor cells re-aggregated into pellets and grafted to $\mathrm{HH} 24$ buds $(\mathbf{f})$ sort out after $24 \mathrm{~h}(\mathbf{g}, \mathbf{h}, n=3 / 3)$ and $48 \mathrm{~h}(\mathbf{i}, \mathbf{j}, n=3 / 3)$. Note the difference in cell distribution between the homochronic and heterochronic mixed grafts was very significantly different (Mann-Whitney-Wilcoxon $U$-test$P$-value $<0.0001$ ). Autopod progenitor cells (then at $\mathrm{HH} 27-\mathbf{g}$ or $\mathrm{HH} 29-\mathbf{i}$ ) predominantly localize to the periphery of the grafts (between the yellow rings) to aggregate with host cells of the same age and zeugopod progenitor cells (then at $\mathrm{HH} 24-\mathbf{g}$ or $\mathrm{HH} 27-\mathbf{i}$ ) are confined to the centre of the grafts. Development of grafts comprising of $\mathrm{HH} 24$ GFP-expressing autopod progenitor cells and wild-type $\mathrm{HH} 20$ zeugopod progenitor cells made to $\mathrm{HH} 24$ buds (k). Autopod progenitor cells (then at day 12) localize to edge of grafted tissue to contact host cells of same age and zeugopod progenitor cells (then at day 11) are confined to the centre of the graft $(\mathbf{I}, \mathbf{m} n=4 / 5)$. Such grafts contribute to the autopod (n,o -purple asterisks $n=5 / 5$; compare with HH2O-20 grafts, blue asterisks; $\mathrm{HH} 24-\mathrm{HH} 24$ grafts, red asterisks and $\mathrm{HH} 20-24$ grafts black asterisks). Note in (n) u-ulna; r-radius; 1,2 and 3 are the digits in anterior to posterior sequence). Scale bars $-100 \mu \mathrm{m}$ in $(\mathbf{b}, \mathbf{d}, \mathbf{g}, \mathbf{i} ; 1 \mathrm{~mm}$ in $\mathbf{I}, \mathbf{n})$. 

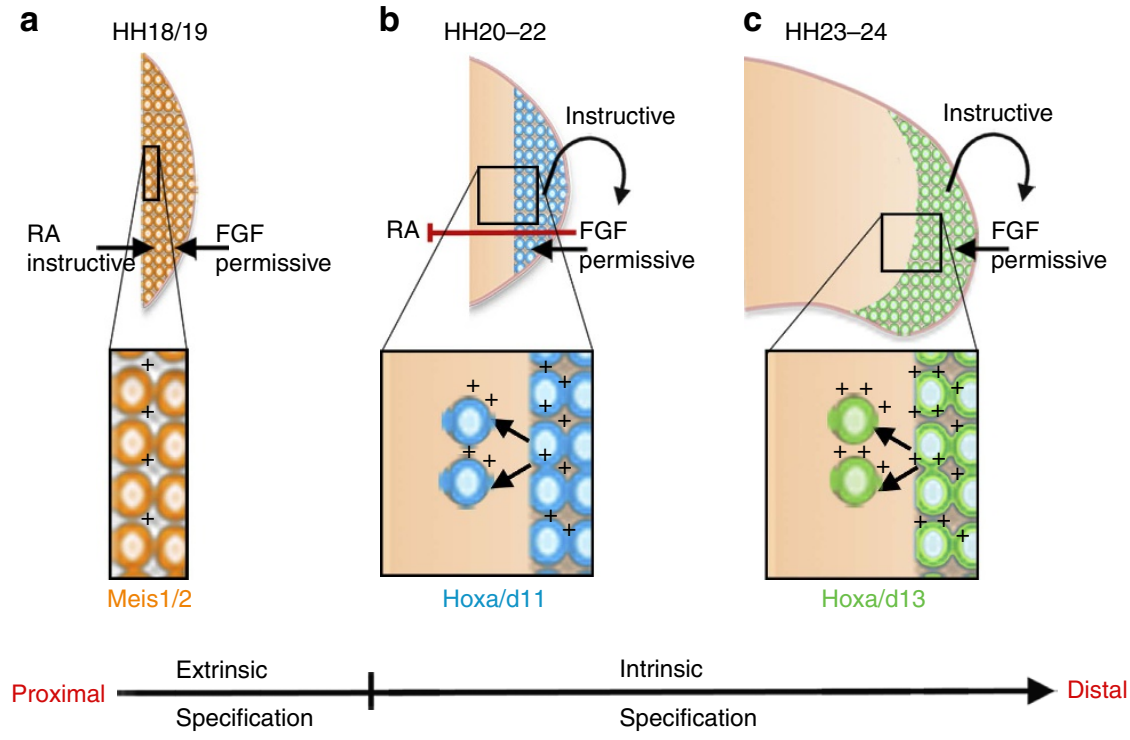

d

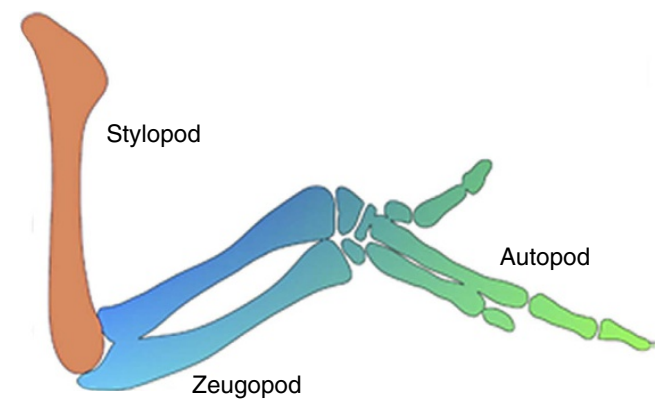

Figure 6 | Model for chick wing proximo-distal patterning. At early limb initiation stages (HH18/19) trunk-derived retinoic acid (RA) specifies the positional value of the stylopod (humerus, $\mathbf{a}, \mathbf{d}$-orange) and then ( $\mathrm{HH} 20-22$ ) intrinsic timing specifies the positional values of the zeugopod (forewing, $\mathbf{b}, \mathbf{d}$ -blue) and later still (HH23-24) the autopod (wrist/digits, $\mathbf{c}, \mathbf{d}$-green). AER-derived FGFs are permissive factors that sustain growth of the limb bud and suppress the proximal programme including Meis1/2 expression by inducing Cyp26b1 that degrades retinoic acid (red line). Elimination of retinoic acid from the distal mesenchyme of the wing bud triggers the switch to intrinsic timing and mesenchyme cells express $5^{\prime} H o x a / d$ genes and maintain AER-derived FGFs. Following the phase of proximal specification (a), cell adhesion properties and hence positional values intrinsically change over time (b,c, greater adhesion of cells shown by additional + symbols in lower insets) and this results in a spatial gradient of positional values along the proximo-distal axis as cells are displaced from the distal mesenchyme by an intrinsic programme of proliferation (arrows-lower insets a-c-graded blue/green on skeleton, $\mathbf{d}$ ).

(Fig. 6b,c). It is possible that the AER and subjacent mesenchyme mutually maintain each other's maturation stage and that the AER reverts to a less mature stage upon our transplantation experiments. The possibility that permissive AER signals increase over time, which has never been demonstrated, cannot be excluded and requires further investigation.

A fundamental issue is whether extrinsic signals or an intrinsic timer re-specifies missing positional values during limb regeneration. It was recently demonstrated that amputated adult axolotl limbs regenerate missing structures in a proximal to distal sequence $^{29}$, rather than by intercalation as previously suggested ${ }^{30}$. Thus, since it is difficult to conceive that signalling gradients can operate over the sheer size of the adult limb, an attractive alternative is that an intrinsic timer, recapitulating the embryonic one we have described here, governs limb regeneration-of at least the zeugopod and autopod segments. On a more general note, it is likely that the timers operating during proximo-distal limb patterning and somitogenesis share common components, such as the Hairy 2 gene, that has an oscillatory expression profile in both systems ${ }^{6,31}$. Moreover, an emerging theme is that both signal and time-based mechanisms operate together during embryogenesis ${ }^{32}$. Whether timing in other patterning systems is an intrinsic property remains largely undetermined.

\section{Methods}

Chick husbandry and tissue grafting. Wild-type and GFP-expressing fertilized Brown Leghorn chicken eggs were incubated, opened and staged according to Hamilton Hamburger ${ }^{33}$. For tissue grafts, wing buds of GFP-expressing HH20 or HH24 embryos were used, the posterior border containing the polarizing region was discarded and a stripe of $150 \mu \mathrm{m}$ of distal sub-AER mesenchyme was dissected The overlying ectoderm was dissected away after incubation in $0.25 \%$ trypsin at room temperature for $2 \mathrm{~min}$ and the mesenchyme stripe was then cut into two or three cuboidal pieces. These were then placed in slits made using a fine sharpened tungsten needle along the junction between the AER and subjacent mesenchyme in the mid-distal region of wing buds of normal host embryos at HH20 or HH24. Hosts were immediately returned to the incubator and harvested for analyses as desired. Before fixation, the development of the graft was examined and photographed under UVA light. For the grafts of re-aggregated distal tissue, stripes of distal tissue of GFP-expressing and wild-type wing buds were obtained following the above protocol. The tissue was disaggregated to single cells by gentle pipetting that were counted to make a ratio of 1 GFP-expressing to seven wild-type cells. Pilot experiments determined this ratio of labelled versus unlabelled cells was optimal for detection of labelled cells. The cells were then centrifuged at 2,500 r.p.m. for $8 \mathrm{~min}$ and resulting pellets were incubated for $20 \mathrm{~min}$ at $38^{\circ} \mathrm{C}$ for consolidation, and then sectioned to generate blocks of $150 \mu \mathrm{m}$ for tissue grafts.

In situ hybridization. Digoxigenin-labelled antisense riboprobes were prepared for Gfp, Fgf8 and Hoxa13. Gfp (Aequorea victoria) is the full-length open reading frame, cloned into the pGEM T easy vector (Promega) and transcribed with SP6 polymerase (Roche); Fgf8 (Gallus gallus) is the full-length open reading frame cloned into pBlueScript and transcribed with T7 polymerase (Roche); Hoxa13 
(Gallus gallus) is a partial clone (80-290 of a 290 amino acid protein) in pBlueScript and transcribed with T3 polymerase (Roche). Briefly, for in situ hybridization in tissue sections, the samples were fixed overnight in 4\% PFA, dehydrated, cleared, embedded in paraffin and sectioned at $7 \mu \mathrm{m}$. Consecutive sections were placed on separate slides to be analysed with different probes. The sections were de-paraffined, rehydrated, mildly digested with proteinase $\mathrm{K}\left(10 \mu \mathrm{g} \mathrm{ml}^{-1}\right.$ for $10 \mathrm{~min}$ ) and hybridized overnight. Sections were then washed with decreasing concentrations of SSC in $50 \%$ formamide (at $65^{\circ} \mathrm{C}$ ), and then blocked at room temperature with $10 \%$ sheep serum before overnight incubation at $4{ }^{\circ} \mathrm{C}$ in the standard anti-digoxigenin antibody conjugated to alkaline phosphatase (Roche,1:2,000). Finally, the staining reaction was carried out with NBT/BCIP and allowed to develop for the desired time.

\section{Immunofluorescence and quantification of cell distribution. Detection of} GFP-expressing cells was performed by immunofluorescence on $7 \mu \mathrm{m}$ paraffin sections. The sections were de-waxed, and blocked for $1-2 \mathrm{~h}$ with blocking buffer ( $1 \%$ bovine serum albumin, $1 \%$ goat serum in phosphate-buffered saline (PBS)). Cells were then incubated overnight at $4{ }^{\circ} \mathrm{C}$ with a rabbit polyclonal antibody for GFP (Life technologies) diluted 1:1,000 in washing buffer (blocking buffer diluted 1:10 in $1 \times$ PBS), and immune complexes were detected with an Alexa-488 conjugated anti-rabbit antibody (Invitrogen) diluted 1:250 in washing buffer (in all cases, fluorescence was only observed in transgenic tissue expressing GFP and not in wild-type tissue). Finally, sections were counterstained with $0.5 \mathrm{mg} \mathrm{ml}^{-1}$ of DAPI, mounted in Vectashield and $1.5 \mu \mathrm{m}$ thick Z-stacks (five optical sections per Z-stack) were acquired using a SP-5 laser-scan confocal microscope (Leica Microsystems) with a $\times 20,0.7 \mathrm{NA}$ objective, a 2 Airy pinhole and $400 \mathrm{~Hz}$ scanning speed. Cells were excited sequentially with 405 and $488 \mathrm{~nm}$ laser lines and fluorescence emission captured between 415 and $480 \mathrm{~nm}$ (DAPI) and 499 and $578 \mathrm{~nm}$ (GFP). Image analyses were performed with Image J: a maximum projection of the Z-stacks was generated after images were median-filtered to reduce noise. First, the perimeter of the graft was used to create an initial region of interest that was reduced by $50 \%$ using the transform tool in Adobe Photoshop to generate an inner region and outer region. Following this, the images were automatically thresholded and the GFP + and DAPI + area within the inner and outer regions of the graft were determined. The GFP + / DAPI + areas were calculated and compared between the two types of graft in both the outer and inner regions. To quantitate the immunofluorescence, three sections from each specimen were analysed. The proportion of the area occupied by the GFP-positive cells in the inner and outer regions of the grafts was determined. The calculation of the $P$-value was performed using three tests of the R software (that is, two $t$-tests with and without the homoscedasticity assumption and the nonparametric Mann-WhitneyWilcoxon $U$-test).

TUNEL-labelling. Detection of apoptosis was performed in sections of paraffin embedded tissue $(7 \mu \mathrm{m})$ using terminal deoxynucleotidyl transferase mediated dUTP nick-end labelling (TUNEL) with the Apoptag Fluorescein Direct In Situ Apoptosis Detection Kit (Intergen) following the manufacturer's instructions.

Flow cytometry. Wild-type and GFP-expressing distal mesenchyme tissue was dissected in ice cold PBS under a LeicaMZ16F UV microscope using a fine surgical knife pooled from replicate experiments (between 10 and 12), and digested into single cell suspensions with trypsin $(0.5 \%$, Gibco) for $30 \mathrm{~min}$ at room temperature. Cells were washed in PBS $(2 \times)$, fixed in $70 \%$ ethanol overnight, washed in PBS $(2 \times)$ and re-suspended in PBS containing $0.1 \%$ Triton X-100,50 $\mu \mathrm{g} \mathrm{ml}{ }^{-1}$ of propidium iodide and $50 \mu \mathrm{g} \mathrm{ml}^{-1}$ of RNase A (Sigma). Dissociated cells were left at room temperature for $20 \mathrm{~min}$, aggregated cells were removed by filtration and the single cells then analysed for DNA content with a FACSCalibur flow cytometer and FlowJo software (Tree star Inc). Based on ploidy values cells were assigned in G1, S or G2/M phases and this was expressed as a percentage of the total cell number (approximately 10,000 in each case). To obtain stage-specific cell cycle profiles between three and six replicate experiments (pools of 10-12 blocks of tissue from separate embryos in each case) were performed and the standard error was calculated. Statistical significance of numbers of G1, S and G2/M phase cells between pools of left and right cubes of distal mesenchyme cells (10-12 in each cases) from the same embryos in homochronic, heterochronic grafting experiments-and also left and right controls was determined by Pearson's $\chi^{2}$ tests to obtain two-tailed $P$-values (significantly different being a $P$-value of $<0.05$ ).

\section{References}

1. Mercader, N. et al. Opposing RA and FGF signals control proximodistal vertebrate limb development through regulation of Meis genes. Development 127, 3961-3970 (2000).

2. Mariani, F. V., Ahn, C. P. \& Martin, G. R. Genetic evidence that FGFs have an instructive role in limb proximal-distal patterning. Nature 453, 401-405 (2008).

3. Rosello-Diez, A., Ros, M. A. \& Torres, M. Diffusible signals, not autonomous mechanisms, determine the main proximodistal limb subdivision. Science 332, 1086-1088 (2011)
4. Cooper, K. L. et al. Initiation of proximal-distal patterning in the vertebrate limb by signals and growth. Science 332, 1083-1086 (2011).

5. Cunningham, T. J. \& Duester, G. Mechanisms of retinoic acid signalling and its roles in organ and limb development. Nat. Rev. Mol. Cell Biol. 16, 110-123 (2015).

6. Pascoal, S. et al. A molecular clock operates during chick autopod proximaldistal outgrowth. J. Mol. Biol. 368, 303-309 (2007).

7. Vargesson, N., Kostakopoulou, K., Drossopoulou, G., Papageorgiou, S. \& Tickle, C. Characterisation of hoxa gene expression in the chick limb bud in response to FGF. Dev. Dyn. 220, 87-90 (2001).

8. Rosello-Diez, A., Arques, C. G., Delgado, I., Giovinazzo, G. \& Torres, M. Diffusible signals and epigenetic timing cooperate in late proximo-distal limb patterning. Development 141, 1534-1543 (2014).

9. Summerbell, D., Lewis, J. H. \& Wolpert, L. Positional information in chick limb morphogenesis. Nature 244, 492-496 (1973).

10. Towers, M., Wolpert, L. \& Tickle, C. Gradients of signalling in the developing limb. Curr. Opin. Cell Biol. 24, 181-187 (2012).

11. Sheeba, C. J., Andrade, R. P. \& Palmeirim, I. Limb patterning: from signaling gradients to molecular oscillations. J. Mol. Biol. 426, 780-784 (2014).

12. McGrew, M. J. et al. Efficient production of germline transgenic chickens using lentiviral vectors. EMBO Rep. 5, 728-733 (2004).

13. Vargesson, N. et al. Cell fate in the chick limb bud and relationship to gene expression. Development 124, 1909-1918 (1997).

14. Sato, K., Koizumi, Y., Takahashi, M., Kuroiwa, A. \& Tamura, K. Specification of cell fate along the proximal-distal axis in the developing chick limb bud. Development 134, 1397-1406 (2007).

15. Lu, P., Yu, Y., Perdue, Y. \& Werb, Z. The apical ectodermal ridge is a timer for generating distal limb progenitors. Development 135, 1395-1405 (2008).

16. Zwilling, E. \& Hansborough, L. Interactions between limb bud ectoderm and mesoderm in the chick embryo III. Experiments with polydactylous limbs. J. Exp. Zool. 132, 219-239 (1956).

17. Zuniga, A., Haramis, A. P., McMahon, A. P. \& Zeller, R. Signal relay by BMP antagonism controls the SHH/FGF4 feedback loop in vertebrate limb buds. Nature 401, 598-602 (1999).

18. Fernandez-Teran, M. \& Ros, M. A. The Apical Ectodermal Ridge: morphological aspects and signaling pathways. Int. J. Dev. Biol. 52, 857-871 (2008).

19. Ganan, Y., Macias, D., Duterque-Coquillaud, M., Ros, M. A. \& Hurle, J. M. Role of TGF beta s and BMPs as signals controlling the position of the digits and the areas of interdigital cell death in the developing chick limb autopod. Development 122, 2349-2357 (1996).

20. Chinnaiya, K., Tickle, C. \& Towers, M. Sonic hedgehog-expressing cells in the developing limb measure time by an intrinsic cell cycle clock. Nat. Commun. 5, 4230 (2014).

21. Nardi, J. B. \& Stocum, D. L. Surface properties of regenerating limb cells: Evidence for gradation along the proximodistal axis. Differentiation 25, 27-31 (1984).

22. Wada, N. \& Ide, H. Sorting out of limb bud cells in monolayer culture. Int. J. Dev. Biol. 38, 351-356 (1994).

23. Ide, H., Wada, N. \& Uchiyama, K. Sorting out of cells from different parts and stages of the chick limb bud. Dev. Biol. 162, 71-76 (1994).

24. Probst, S. et al. SHH propagates distal limb bud development by enhancing CYP26B1-mediated retinoic acid clearance via AER-FGF signalling. Development 138, 1913-1923 (2011).

25. Lewis, J. H. Fate maps and the pattern of cell division: a calculation for the chick wing-bud. J. Embryol. Exp. Morphol. 33, 419-434 (1975).

26. Stadler, H. S., Higgins, K. M. \& Capecchi, M. R. Loss of Eph-receptor expression correlates with loss of cell adhesion and chondrogenic capacity in Hoxa13 mutant limbs. Development 128, 4177-4188 (2001).

27. Sheth, R. et al. Hox genes regulate digit patterning by controlling the wavelength of a Turing-type mechanism. Science 338, 1476-1480 (2012).

28. Rubin, L. \& Saunders, Jr. J. W. Ectodermal-mesodermal interactions in the growth of limb buds in the chick embryo: constancy and temporal limits of the ectodermal induction. Dev. Biol. 28, 94-112 (1972).

29. Roensch, K., Tazaki, A., Chara, O. \& Tanaka, E. M. Progressive specification rather than intercalation of segments during limb regeneration. Science 342, 1375-1379 (2013).

30. French, V., Bryant, P. J. \& Bryant, S. V. Pattern regulation in epimorphic fields. Science 193, 969-981 (1976).

31. Palmeirim, I., Henrique, D., Ish-Horowicz, D. \& Pourquie, O. Avian hairy gene expression identifies a molecular clock linked to vertebrate segmentation and somitogenesis. Cell 91, 639-648 (1997).

32. Dessaud, E. et al. Interpretation of the sonic hedgehog morphogen gradient by a temporal adaptation mechanism. Nature 450, 717-720 (2007).

33. Hamburger, V. \& Hamilton, H. L. A series of normal stages in the development of the chick embryo. 1951. J. Morphol. 88, 49-92 (1951). 


\section{Acknowledgements}

M.T. and K.C. thank the Medical Research Council. M.A.R., I.D. and P.S-L. thank the Spanish Ministry of Economy and Innovation (BFU2011-24972). We thank Cheryll Tickle for critical reading and hosting I.D. and Susan Mackem for critical reading, Marisa Junco for technical support, Susan Clark for flow cytometry and Adrian Sherman and Helen Sang for GFP-expressing chicken embryos and allowing M.A.R. to establish a flock. P.S-L. was supported by a CSIC JAE-Pre contract co-funded by the FSE.

\section{Author contributions}

P.S-L. and M.A.R. grafted normal and re-aggregated distal tissue and performed in situ hybridizations and TUNEL analyses. K.C. grafted normal distal tissue and did flow cytometry. V.M.C. performed and quantified immunofluorescence experiments and I.D. grafted normal distal tissue and performed pilot experiments. M.T. and M.A.R. designed the experiments, all authors discussed the results, M.T. wrote the paper and M.A.R. edited the paper.

\section{Additional information}

Supplementary Information accompanies this paper at http://www.nature.com/ naturecommunications

Competing financial interests: The authors declare no competing financial interests.

Reprints and permission information is available online at http://npg.nature.com/ reprintsandpermissions/

How to cite this article: Saiz-Lopez, P. et al. An intrinsic timer specifies distal structures of the vertebrate limb. Nat. Commun. 6:8108 doi: 10.1038/ncomms9108 (2015).

\section{(c) (i)}

This work is licensed under a Creative Commons Attribution 4.0 International License. The images or other third party material in this article are included in the article's Creative Commons license, unless indicated otherwise in the credit line; if the material is not included under the Creative Commons license, users will need to obtain permission from the license holder to reproduce the material. To view a copy of this license, visit http://creativecommons.org/licenses/by/4.0/ 